\title{
DEPENDENCE OF REFLECTED IMPEDANCE ON THE SPEED OF THE RELATIVE MOVEMENT OF THE COIL AND CONDUCTING HALF-SPACE IN EDDY CURRENT MEASUREMENTS
}

\author{
A. N. Pechenkov*, V. E. Shcherbinin \\ M.N. Miheev Institute of Metal Physics of Ural Branch of Russian Academy of Sciences, 18 S. Kovalevskoy st., \\ Ekaterinburg, Russian Federation \\ *Corresponding author. E-mail: Pechenkov@imp.uran.ru; address for correspondence: 18, ul. S. Kovalevskoy, 620990, \\ Ekaterinburg, Russian Federation. Tel.: +7 3433783735
}

Graphic dependences of real and imaginary components of the impedance introduced into the coil on the coil movement speed are constructed. The pictures allow us to estimate the possibilities of eddy current control at high speed of the movement.

Keywords: eddy current, NDT method, computer modeling.

DOI: $10.17804 / 2410-9908.2015 .5 .016-021$

\section{References}

1. Vlasov V.V. Skorostnaya electromagnitnaya defektoskopiya zheleznodorozhnykh relsov. Avtoref. disser. dokt.t.n. [Express Electromagnetic Nondestructive Testing of Railway Track Rails], Tomsk, 1961. (In Russian).

2. Vorobyov A.I. Issledovaniya po skorostnoi defektoskopii relsov zhelesnodorozhnogo puti. Avtoref. disser. k.t.n. [A Research on Express Nondestructive Testing of Railway Track Rails], M., TSNII MPS Publ., 1966. (In Russian).

3. Halileev P.A. Birth of Nondestructive Testing. Defektoskopiya, 1999, no. 2, pp. 73-82. (In Russian).

4. Korolev M.Yu. On the maximum speed of the magnetic testing of rails. V mire nerazrushayushchego kontrolya, 2001, no. 3, p. 13. (In Russian).

5. Gyngazova G.S., Milyaev D.V., Yermoshin N.I. Developing a device for measuring the length of extended nonmagnetic products by eddy-current technique. Vestnik Nauki Sibiri, 2013, no. 1 (7), pp. 117-128. (In Russian).

6. Gerasimov V.G., Klyuev V.V., Sukhorukov V.V. Fedosenko Yu.K. Vikhretokovye metody $i$ sredstva kontrolya. Spravochnik [Eddy-Current Testing Methods and Devices. Handbook]. M., Mashinostroenie Publ., 1995. (In Russian).

7. Diakin V.V., Sandovsky V.A. Teoriya i raschet nakladnykh vikhretokovykh preobrazovatelei [Theory and Design of Attached Eddy-Current Transducers]. M., Nauka Publ., 1981. (In Russian). 
Подана в журнал: 18.09.2015 г.

УДК 620.179 .14

DOI: $10.17804 / 2410-9908.2015 .5 .016-021$

\title{
ЗАВИСИМОСТЬ ВНОСИМОГО ИМПЕДАНСА ОТ СКОРОСТИ ДВИЖЕНИЯ КА- ТУШКИ ОТНОСИТЕЛЬНО ПРОВОДЯЩЕГО ПОЛУПРОСТРАНСТВА ПРИ ВИХРЕТОКОВЫХ ИЗМЕРЕНИЯХ
}

\author{
А. Н. Печенков*, В. Е. Щербинин \\ Федеральное государственное бюджетное учреждение науки Институт физики металлов имени \\ М.Н. Михеева Уральского отделения Российской академии наук, ул. С. Ковалевской, 18, Екатеринбург, \\ Российская Федерация \\ *Ответственный автор. Электронная почта: Pechenkov@imp.uran.ru; адрес для переписки: 620990, \\ ул. С. Ковалевской, 18, Екатеринбург, Российская Федерация. Телефон: +7 (343) 378-37-35
}

Построены наглядные графические зависимости вещественной и мнимой составляющих импеданса, вносимого в катушку, от скорости движения катушки. Полученные графики позволяют оценить возможности вихретокового контроля при высоких скоростях движения. вание.

Ключевые слова: вихревые токи, неразрушающий контроль, компьютерное моделиро-

\section{1. Введение}

В настоящее время одной из важных задач является создание дефектоскопов, основанных на различных физических принципах и позволяющих производить контроль при высоких скоростях движения относительно контролируемого изделия [1-5].

В частности, это относится к вихретоковым приборам. Для вихретокового способа контроля давно известны точные формулы зависимости импеданса катушки от скорости движения для некоторых простейших постановок задачи. Например, для случая движения витка с током над проводящим, намагничивающимся полупространством $[6,7]$. Эти формулы были использованы авторами для построения графических годографов, которые довольно трудно использовать для анализа зависимости результатов измерений от скорости движения. В данной работе для этого случая построены простые и наглядные графики зависимостей вещественной и мнимой составляющих импеданса от скорости движения. Дан физический анализ полученных результатов.

\section{2. Постановка задачи}

Воспользуемся результатами работы [7] для импеданса, вносимого в измерительную катушку полупространством. По определению:

$$
Z=-\frac{E}{I} ; \quad E=\oint_{r=R} e d l ; \quad e=\operatorname{Re}\left[e^{*}(r) \exp j \varpi t\right]
$$

где: Е - э.д.с. на контуре измерительного витка радиуса R; I - величина тока в витке; $\omega$ - угловая частота сигнала. 


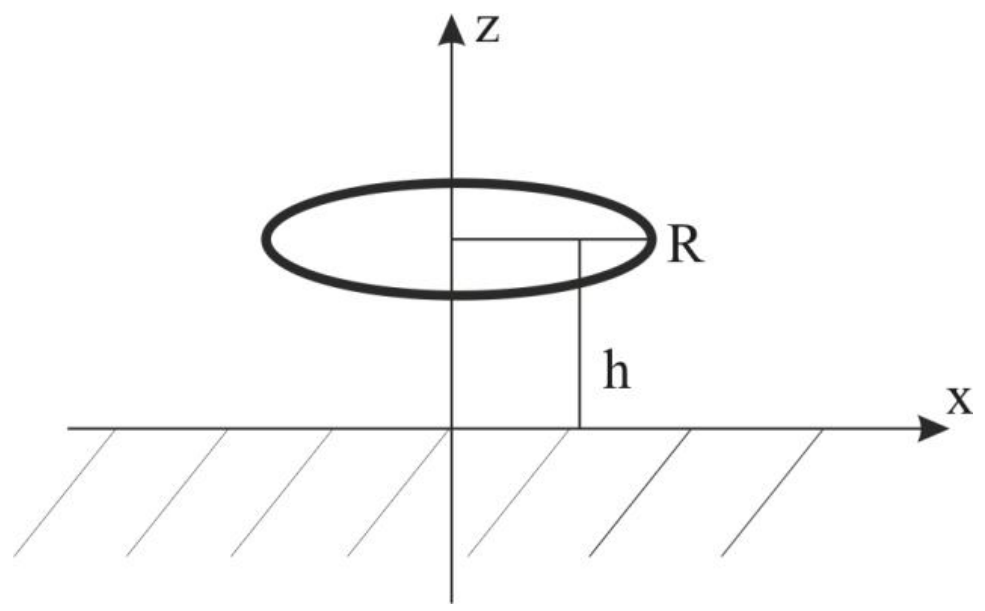

Рис. 1. Виток с током над проводящим намагничивающимся полупространством Формула для вносимого импеданса имеет вид:

$$
Z=\frac{j \varpi \mu_{0} R^{2}}{2} \int_{0}^{\infty} d \lambda J_{1}^{2}(\lambda R) \exp (-2 h \lambda) \int_{0}^{2 \pi} \frac{\mu \lambda-\sqrt{\lambda^{2}+j \mu \mu_{0} \sigma(\varpi-\lambda V \cos \phi)}}{\mu \lambda+\sqrt{\lambda^{2}+j \mu \mu_{0} \sigma(\varpi-\lambda V \cos \phi)}} d \phi
$$

Здесь: $\mathrm{J}_{1}$ - функция Бесселя первого рода; $\mu$ - магнитная проницаемость полупространства; $\sigma$ - электропроводность полупространства; V - скорость движения витка относительно полупространства.

\section{3. Результаты и обсуждение}

Произведём простое, но громоздкое вычисление вещественной и мнимой составляющих импеданса. Обозначим:

$$
\begin{aligned}
& a_{1}=\mu \mu_{0} \sigma(\varpi-\lambda V \cos \phi) ; \quad \sqrt{\lambda^{2}+j a_{1}}=a+j b ; \quad a=\sqrt{\frac{\lambda^{2}}{2}+\frac{1}{2} \sqrt{\lambda^{4}+a_{1}^{2}}} \\
& b=\frac{a_{1}}{2 a} ; \\
& b_{1}=\left(\mu^{2}-1\right) \lambda^{2} ; \quad b_{2}=b_{3}+j b_{4} ; \quad b_{3}=\left(\mu^{2}+1\right) \lambda^{2}-2 \mu \lambda a ; \quad b_{4}=a_{1}-2 \mu \lambda b ; \\
& b_{5}=b_{1} b_{3}-a_{1} b_{4} ; \quad b_{6}=b_{1} b_{4}+a_{1} b_{3} .
\end{aligned}
$$

Тогда можно получить

$$
\begin{aligned}
& \operatorname{Re} Z=-\frac{\varpi \mu_{0} R^{2}}{2} \int_{0}^{\infty} d \lambda J_{1}^{2}(\lambda R) \exp (-2 h \lambda) \int_{0}^{2 \pi} \frac{b_{6}}{b_{1}^{2}+a_{1}^{2}} d \phi \\
& \operatorname{Im} Z=\frac{\varpi \mu_{0} R^{2}}{2} \int_{0}^{\infty} d \lambda J_{1}^{2}(\lambda R) \exp (-2 h \lambda) \int_{0}^{2 \pi} \frac{b_{5}}{b_{1}^{2}+a_{1}^{2}} d \phi
\end{aligned}
$$

Напомним смысл рассмотрения реальной и мнимой частей импеданса. Пусть вещественные амплитуды тока и э.д.с. в витке связаны формулой, которая учитывает сдвиг фаз:

$$
Z=\frac{e}{i} \exp (j \varphi)
$$

Тогда вещественное сопротивление витка и сдвиг фаз можно найти так: 


$$
\begin{aligned}
& \operatorname{Re} Z=\frac{e}{i} \cos (\varphi) ; \operatorname{Im} Z=\frac{e}{i} \sin (\varphi) ; \\
& r=\frac{e}{i}=\sqrt{(\operatorname{Re} Z)^{2}+(\operatorname{Im} Z)^{2}} ; \quad \operatorname{tg}(\varphi)=\frac{\operatorname{Im} Z}{\operatorname{Re} Z} .
\end{aligned}
$$

Для расчётов по формулам (4) были взяты следующие параметры: $\sigma=15 \mathrm{MCM} / \mathrm{M}$; $\mu=100$. Геометрические размеры: $\mathrm{R}=0,1 \mathrm{M} ; \mathrm{h}=0,05$ м. Графики строились для частот: 10 Гц, 100 Гц и 1000 Гц. На рис.2 приведены графики для всех указанных случаев.
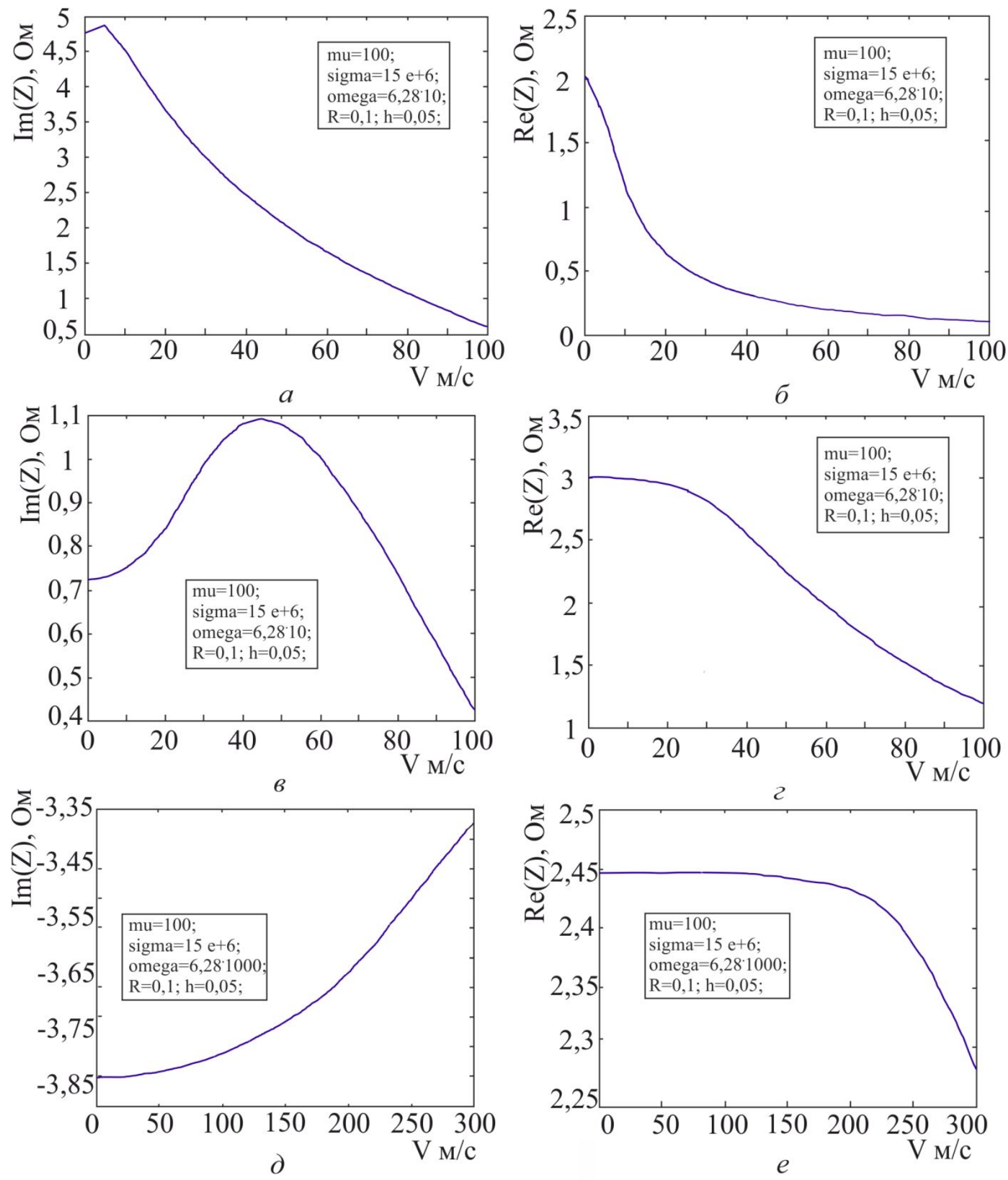

Рис. 2. Графики зависимости реальной и мнимой составляющих импеданса от скорости движения витка для различных значений параметров

Pechenkov A. N. et al. / Dependence of reflected impedance on the speed of the relative movement of the coil and conducting half-space in eddy current measurements. 
open-access journal

\section{4. Заключение}

Из анализа графиков на рис. 2 следует, что с ростом скорости движения вносимый импеданс уменьшается. Однако с ростом частоты границы спада сдвигаются в сторону высоких скоростей. В целом, для реально достижимых скоростей, например железнодорожного транспорта, можно подобрать частоты, при которых эти скорости практически не сказываются на величине вносимого импеданса.

Поясним спад вносимого импеданса на качественном примере. Известно, что индуктированные токи создают магнитное поле, которое уменьшает исходное магнитное поле (закон Ленца). Нужно оценить влияние скорости изменения исходного поля на этот процесс.

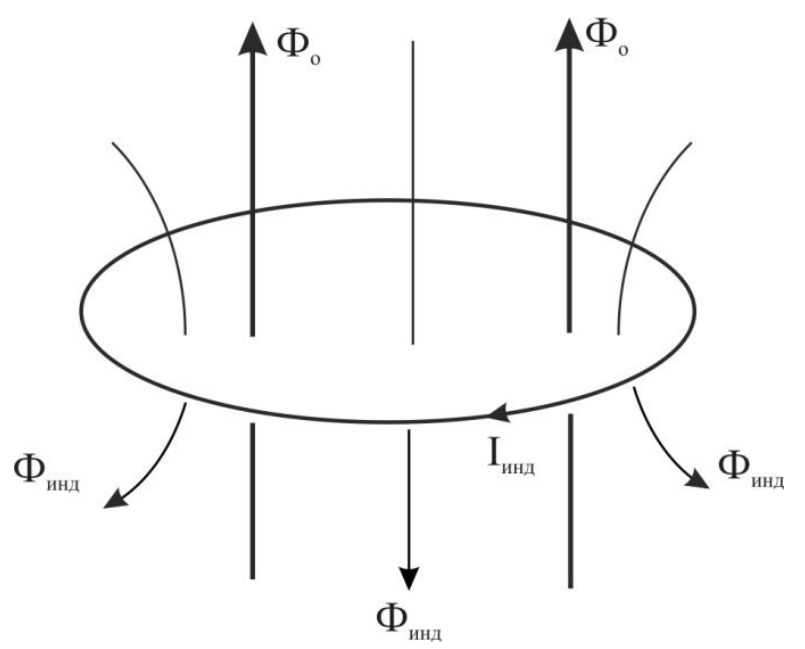

Рис. 3. Контур с индуцированным током

Пусть задан исходный магнитный поток $\Phi_{0}=A_{0} e^{b t}$ через проводящий контур. Коэффициент $b$ определяет скорость изменения потока. При изменении магнитного потока в контуре наводится индукционный ток $\mathrm{I}_{\text {инд }}$, магнитное поле которого создаёт поток $\Phi_{\text {инд }}=A_{\text {инд }} e^{b \mathrm{t}}$, направленный против исходного поля. Пусть далее, индукционные электродвижущая сила и ток связаны линейно: $\varepsilon_{\text {инд }}=\mathrm{RI}_{\text {инд }}$. Тогда, по закону электромагнитной индукции, имеем:

$$
I_{\text {инд }}=\frac{1}{\mathrm{R}} \frac{d\left(\Phi_{0}-\Phi_{\text {инд }}\right)}{\mathrm{dt}} .
$$

Пусть $\Phi_{\text {инд }}=\mathrm{LI}_{\text {инд }}$. Здесь $\mathrm{R}$ - сопротивление контура; $\mathrm{L}$ - индуктивность контура.

Из (7) получаем формулу для неизвестной амплитуды индуцированного магнитного потока:

$$
A_{\text {инд }}=A_{0} \frac{{ }^{в} \mathrm{~L}}{\mathrm{R}+6 \mathrm{~L}} .
$$

Тогда полный магнитный поток через контур будет равен:

$$
\Phi=\Phi_{0}-\Phi_{u н d}=\Phi_{0} \frac{\mathrm{R}}{\mathrm{R}+{ }_{6} \mathrm{~L}} .
$$


Из (9) видно, что при увеличении скорости изменения исходного потока $(b \rightarrow \infty)$ полный поток, во-первых, существенно меньше исходного, а во-вторых, его изменение замедлятся, так как $\frac{d \Phi}{d t}=\frac{d \Phi_{0}}{d t} \frac{\mathrm{R}}{\mathrm{R}+6 \mathrm{~L}}$.

\section{Благодарность}

Работа выполнена по проекту фундаментальных исследований УрО РАН 2015-2017 гг., номер 15-17-2-54.

\section{Литература}

1. Власов В. В. Скоростная электромагнитная дефектоскопия железнодорожных рельсов: автореф. дис. ... д-р техн. наук. - Томск, 1961.

2. Воробьев А. И. Исследования по скоростной дефектоскопии рельсов железнодорожного пути: автореф. дис. ... канд. техн. наук. - М.: ЦНИИ МПС. - 1966.

3. Халилеев П. А. Рождение рельсовой дефектоскопии // Дефектоскопия. - 1999. - № 12. - C. $73-82$.

4. Королев М. Ю. О предельной скорости контроля рельсов магнитным методом // В мире неразрушающего контроля. - 2001. - № 3. - С. 13.

5. Гынгазова Г. С., Миляев Д. В., Ермошин Н. И. Разработка измерителя длины немагнитных протяженных изделий вихретоковым методом // Вестник науки Сибири. - 2013. № 1 (7). - С. 117-128.

6. Вихретоковые методы и средства контроля. Справочник / В. Г. Герасимов, В. В. Клюев, В. В. Сухоруков, Ю. К. Федосенко - М. : Машиностроение, 1995. - С. 269-312. 7. Дьякин В. В., Сандовский В. А. Теория и расчёт накладных вихретоковых преобразователей. - М. : Наука, 1981. - С. 110. 